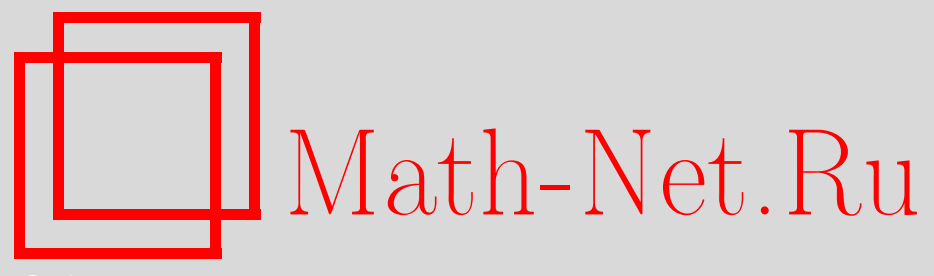

С. В. Гальцев, А. И. Шафаревич, Спектр и псевдоспектр несамосопряженного оператора Шрёдингера с периодическими коэффициентами, Матем. заметки, 2006, том 80, выпуск 3, 356-366

DOI: https://doi.org/10.4213/mzm2821

Использование Общероссийского математического портала Math-Net.Ru подразумевает, что вы прочитали и согласны с пользовательским соглашением http://www . mathnet.ru/rus/agreement

Параметры загрузки:

IP: 54.166 .219 .16

26 апреля 2023 г., 18:37:09

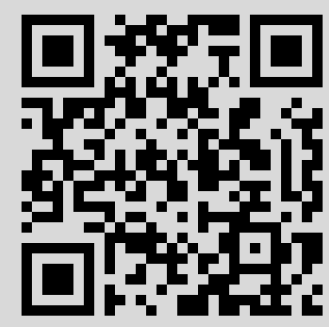




\section{СПЕКТР И ПСЕВДОСПЕКТР \\ НЕСАМОСОПРЯЖЕННОГО ОПЕРАТОРА ШРЁДИНГЕРА \\ С ПЕРИОДИЧЕСКИМИ КОЭФФИЦИЕНТАМИ}

\section{С. В. Гальцев, А. И. Шафаревич}

В работе изучается псевдоспектр несамосопряженного оператора

$$
\mathfrak{D}=-h^{2} \frac{d^{2}}{d x^{2}}+i V(x),
$$

где $V(x)$ - периодичная целая аналитическая функция, действительная на действительной оси, с вещественным периодом $T$. В этом операторе $h$ рассматривается как малый параметр. Показывается, что псевдоспектром данного оператора является замыкание его числового образа - полуполоса в $\mathbb{C}$. Причем псевдособственные функции, т.е. функции $\varphi(h, x)$, удовлетворяющие условию

$$
\|\mathfrak{D} \varphi-\lambda \varphi\|=O\left(h^{N}\right), \quad\|\varphi\|=1, \quad N \in \mathbb{N},
$$

строятся явно. Таким образом, как оказывается, псевдоспектр изучаемого оператора гораздо шире его спектра.

Библиография: 8 названий.

1. Введение. Известно, что структура спектра несамосопряженного дифференциального оператора, как правило, существенно сложнее, чем в самосопряженном случае; в частности, точки спектра могут быть по-разному расположены на комплексной плоскости.

Для самосопряженных задач один из эффективных методов исследования спектра - квазиклассическая асимптотика, позволяющая при определенных условиях вычислять так называемые спектральные серии - наборы чисел, приближенно (вместе с соответствующими функциями) удовлетворяющие спектральному уравнению для исходного оператора. Каждая спектральная серия соответствует изотропному многообразию в фазовом пространстве соответствующей классической системы и вычисляется при помощи так называемых условий квантования Бора-Зоммерфельда-Маслова; если многообразие лагранжево, эти условия имеют вид

$$
\frac{1}{2 \pi h} \int_{\gamma} \Theta=m(\gamma)+\frac{1}{4} \mu(\gamma),
$$

где $\gamma$ - произвольный цикл на многообразии, $\Theta$ - первообразная симплектической структуры, $m(\gamma)$ - произвольное целое число, $\mu(\gamma)$ - индекс Маслова и $h \rightarrow 0$ - квазиклассический параметр. Условия квантования должны быть выполнены для всех

(С) С. В. ГАЛЬЦЕв, А.И. ШАФАРЕВич, 2006 
циклов на лагранжевом многообразии; они необходимы для существования на этом многообразии канонического оператора Маслова, определяющего асимптотическую собственную функцию. Самосопряженность исходного оператора гарантирует близость спектральной серии к спектру исходной задачи: если спектральное уравнение выполнено $\bmod O\left(h^{N}\right)$, то в $h^{N}$-окрестности любой точки спектральной серии найдется точка спектра.

Развитой квазиклассической теории, описывающей спектры несамосопряженных операторов, к настоящему времени не существует; это связано, во-первых, с тем, что соответствующие изотропные многообразия, как правило, оказываются комплексными, а во-вторых, с тем, что спектральные серии могут не иметь никакого отношения к спектру исходного оператора. В работах [1], [2] исследовался так называемый псевдоспектр - множество, состоящее из чисел, приближенно удовлетворяющих спектральному уравнению; в частности, отмечалось различие между псевдоспектром и асимптотикой точного спектра. В [3]-[6] асимптотика спектра вычислена для операторов Шрёдингера и Орра-Зоммерфельда, заданных на отрезке; в частности, показано, что точки спектра асимптотически стремятся к некоторому графу на комплексной плоскости, в то время как псевдоспектр может заполнять целые области.

В настоящей работе изучается спектр и псевдоспектр оператора $-h^{2} \partial^{2} / \partial x^{2}+$ $i V(x)$, где $V$ - периодическая функция, вещественная на вещественной оси (например, $V=\cos x)$. Показано, что при $h \rightarrow 0 \quad h^{N}$-псевдоспектр этого оператора для любого $N$ заполняет полуполосу на комплексной плоскости, в то время как настоящий спектр $\bmod O\left(h^{2}\right)$ концентрируется вблизи одномерного множества (графа). Ребра этого графа соответствуют различным спектральным сериям, которые могут быть вычислены при помощи условий Бора-Зоммерфельда-Маслова на комплексной кривой (римановой поверхности); в отличие от самосопряженного случая разные циклы на одной и той же поверхности определяют разные серии (другими словами, для конструкции асимптотики достаточно требовать выполнения условий квантования только на одном цикле).

2. Постановка задачи. Ряд вопросов, естественно возникающих в спектральной теории дифференциальных операторов, приводит к исследованию спектра оператора

$$
\mathfrak{D}=-h^{2} \frac{d^{2}}{d x^{2}}+i V(x),
$$

где $V(x)$ - периодичная целая аналитическая функция, действительная на действительной оси, с вещественным периодом T. В частности, (1) возникает как "эталонный" оператор в теории гидродинамической устойчивости: его спектр при определенных условиях похож на спектр оператора Орра-Зоммерфельда. Другой пример - спектральная задача для оператора $\varepsilon \Delta+(v(x), \Delta)$ на плоском торе (здесь $x=$ $\left.\left(x_{1}, x_{2}\right) \in \mathbb{T}^{2}\right)$. Пусть $v(x)$ - бездивергентное поле вида $v\left(x_{1}, x_{2}\right)=w\left(x_{1}\right) \partial / \partial x_{2}$. Спектральная задача допускает разделение переменных: собственная функция $\varphi\left(x_{1}, x_{2}\right)$ имеет вид $e^{i m x_{2}} \psi\left(x_{1}\right)$, причем $\psi$ удовлетворяет спектральной задаче для оператора (1) при $V\left(x_{1}\right)=m w\left(x_{1}\right)$ и $\varepsilon=h^{2}$.

Оператор $\mathfrak{D}$ естественно рассматривать как неограниченный несамосопряженный оператор в $L_{2}\left(\mathbb{S}^{1}=\mathbb{R} / T \mathbb{Z}\right)$, в то же время, поскольку уравнение $(\mathfrak{D}-E) \varphi=0$ - обыкновенное линейное дифференциальное уравнение с целыми аналитическими коэф- 
фициентами, все его решения - аналитические функции, и $\mathfrak{D}$ можно рассматривать как оператор в пространстве $\mathscr{A}(\mathbb{C} / T \mathbb{Z})$ целых аналитических периодических функций. Цель настоящей работы - сравнение асимптотики при $h \rightarrow 0$ спектра оператора $\mathfrak{D}$ и псевдоспектра этого оператора в частном случае $V(x)=\cos x$.

Подробному описанию асимптотики спектра посвящена отдельная статья авторов [7]. Оказывается, эта асимптотика спектра выражается через интегралы от голоморфных форм по циклам на римановой поверхности $\Lambda$, задаваемой в $\mathbb{C}^{2} / T \mathbb{Z}$ уравнением $p^{2}+i V(x)=E \quad\left(p \in \mathbb{C}, x \in \mathbb{C}^{2} / T \mathbb{Z}\right)$. Эта (некомпактная) поверхность при $E \neq \pm i$ гомеоморфна сфере с четырьмя дырками и получается склейкой двух экземпляров цилиндра $\mathbb{C} / T \mathbb{Z}$ по отрезку, соединяющему нули функции $i V(x)-E$. Именно, справедлива следующая

Теорема 1. Пусть Е таково, что на поверхности $\Lambda$ существует иикл $\gamma, \partial л я$ которого

$$
\frac{1}{2 \pi h} \int_{\gamma} p d x=m+\frac{\mu}{2} .
$$

Тогда существует собственное число $\lambda$ оператора $\mathfrak{D}$, для которого $\lambda-E=O\left(h^{2}\right)$. Здесь $\mu=0$, если чикл $\gamma$ становится стягиваемым при заклеивании некоторой дырки на $\Lambda, u \mu=1$ в противоположном случае.

На сфере с четырьмя дырками имеется три базисных цикла. Тем самым выделяется три серии собственных чисел - каждая соответствует базисному циклу на поверхности $\Lambda$. При $h \rightarrow 0$ собственные значения концентрируются в $O\left(h^{2}\right)$ - окрестности графа на комплексной плоскости. Ребра этого графа задаются уравнениями

$$
\frac{1}{2 \pi h} \int_{\gamma_{j}} p d x=0 .
$$

Назовем этот граф пределъным спектральным графом оператора $\mathfrak{D}$. Вне окрестностей точек $\pm i$ предельный спектральный граф выглядит так, как показано на рис. 1.

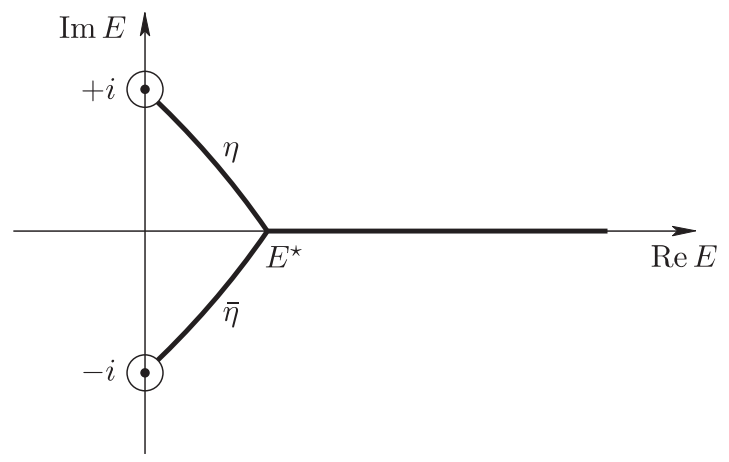

Рис. 1

В этой работе мы найдем псевдоспектр оператора $\mathfrak{D}$ и покажем, что он существенно больше спектра. 
3. Общие определения и формулировка основного результата. Пусть задано пространство функций, снабженное скалярным произведением

$$
\Phi=(\{\varphi(x)\},(\cdot, \cdot)) .
$$

Обозначим через $\|\cdot\|$ норму на этом пространстве, порожденную этим скалярным произведением. Кроме того, пусть задан линейный (неограниченный) оператор

$$
A=A(x, \varepsilon): \Phi_{0} \rightarrow \Phi, \quad \overline{\Phi_{0}}=\Phi,
$$

зависящий от параметра $\varepsilon \in(0,+\infty)$.

ОпредЕЛЕНиЕ 1. Точка $\lambda$ принадлежит $\varepsilon$-nсевдоспектру оператора $A=A(x, \varepsilon)$ тогда и только тогда, когда найдется функция $\varphi=\varphi(x, \varepsilon)$, принадлежащая единичной сфере пространства $\Phi_{0}$ (т.е. $\|\varphi(\cdot, \varepsilon)\|=1$ ) для любого фиксированного $\varepsilon \in(0,+\infty)$ и обладающая свойством

$$
\|A(x, \varepsilon) \varphi(x, \varepsilon)-\lambda \varphi(x, \varepsilon)\|=O(\varepsilon) \quad \text { при } \quad \varepsilon \rightarrow 0+0
$$

$\varepsilon$-псевдоспектр оператора $A$ будем обозначать $\operatorname{PSP}_{\varepsilon}(A)$.

ЗАмечАние 1 . Отметим, что $\varepsilon$-псевдоспектр не зависит от $\varepsilon$, а зависит только от того, как параметр $\varepsilon$ входит в оператор $A(x, \varepsilon)$.

Основной результат настоящей работы теперь можно сформулировать так.

Tеорема 2. В случае непостоянной $V(x)$ для любого $N$ из натуралъного ряда $h^{N}$-псевдоспектр оператора $-h^{2} d^{2} / d x^{2}+i V(x)$, заданного на окружности, равен полуполосе $[0,+\infty)+i[\min V, \max V]$.

То есть псевдоспектр выглядит так, как показано на рис. 2.

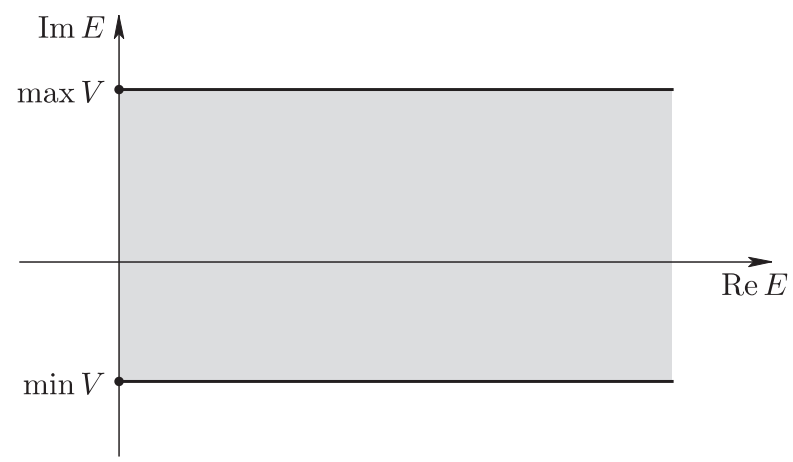

Рис. 2

При сравнении с теоремой 1 видим насколько сильно отличается спектр от псевдоспектра.

\section{4. Связь псевдоспектра и числового образа.}

ЛЕмма 1. в-псевдоспектр замкнут. 
ДокАЗАТЕЛЬСтво. Рассмотрим произвольную точку из замыкания $\varepsilon$-псевдоспектра: $\lambda_{0} \in \overline{\operatorname{PSP}_{\varepsilon}(A)}$. Тогда по определению замыкания имеем

$$
(\forall \delta>0)\left(\exists \lambda_{\delta} \in \operatorname{PSP}_{\varepsilon}(A)\right):\left|\lambda_{0}-\lambda_{\delta}\right|<\delta .
$$

Это верно в том числе для $\delta=\varepsilon$. Кроме того, по определению $\varepsilon$-псевдоспектра

$$
\exists \varphi(x, \varepsilon) \in\left\{\varphi(\cdot, \varepsilon) \in \Phi_{0} \mid\|\varphi(\cdot, \varepsilon)\|=1\right\}:\left\|A \varphi-\lambda_{\delta} \varphi\right\|=O(\varepsilon) .
$$

Беря $\delta=\varepsilon$ и объединяя написанное выше, получаем

$$
\begin{aligned}
0 \leqslant\left\|A \varphi-\lambda_{0} \varphi\right\| & =\left\|A \varphi-\lambda_{\varepsilon} \varphi+\left(\lambda_{\varepsilon}-\lambda_{0}\right) \varphi\right\| \\
& \leqslant\left\|A \varphi-\lambda_{\varepsilon} \varphi\right\|+\left|\lambda_{\varepsilon}-\lambda_{0}\right| \cdot\|\varphi\|<O(\varepsilon)+\varepsilon \cdot 1=O(\varepsilon) .
\end{aligned}
$$

Значит, $\left\|A \varphi-\lambda_{0} \varphi\right\|=O(\varepsilon)$; следовательно, $\lambda_{0}$ - точка $\varepsilon$-псевдоспектра. Другими словами, $\overline{\operatorname{PSP}_{\varepsilon}(A)} \subseteq \operatorname{PSP}_{\varepsilon}(A)$; значит, $\varepsilon$-псевдоспектр замкнут.

ОПредЕЛЕНИЕ 2. Числовым образом оператора $A$ называется множество

$$
\left\{(A \psi, \psi) \mid \psi \in \Phi_{0},\|\psi\|=1\right\} .
$$

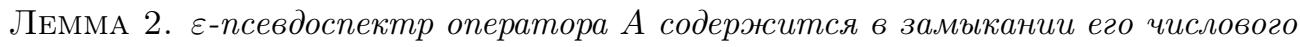
образа.

ДокАЗАТЕЛьство. Действительно, пусть $E$ - точка $\varepsilon$-псевдоспектра; тогда найдется функция $\psi,\|\psi\|=1$, удовлетворяющая равенству $A \psi=E \psi+O(\varepsilon)$. Умножим скалярно это равенство на $\psi$ :

$$
(A \psi, \psi)=(E \psi, \psi)+(O(\varepsilon), \psi)
$$

С учетом того, что $(\psi, \psi)=\|\psi\|=1$ и $(O(\varepsilon), \psi)=O(\varepsilon)$, получаем

$$
(A \psi, \psi)=E+O(\varepsilon) .
$$

Другими словами, $E \in\left\{(A \psi, \psi) \mid \psi \in \Psi_{0},\|\psi\|=1\right\}+O(\varepsilon)$. Устремляя $\varepsilon$ к нулю, получаем требуемое:

$$
E \in \overline{\left\{(A \psi, \psi) \mid \psi \in \Psi_{0},\|\psi\|=1\right\}}
$$

СЛЕДСТвиЕ 1. Пусть $\varepsilon(h)$ - непрерывная обратимая функиия параметра $h u$ $\varepsilon(0+0)=0+0$. Тогда $\varepsilon(h)$-псевдоспектр оператора $\mathfrak{D}$ содержится в полуполосе $[0,+\infty)+i[\min V, \max V]$.

Доказательство. В нашем случае $\Phi=L_{2}\left(\mathbb{S}^{1}\right), \Phi_{0}=W_{2}^{2}\left(\mathbb{S}^{1}\right), A(x, \varepsilon)=D_{\mathbb{S}^{1}}=$ $-h(\varepsilon)^{2} d^{2} / d x^{2}+i V(x)$. Скалярное произведение на пространстве $L_{2}\left(\mathbb{S}^{1}\right)$ задается формулой

$$
(u, v)=\int_{\mathbb{S}^{1}} u \bar{v} d x .
$$

Распишем выражение $\left(D_{\mathbb{S}^{1}} \psi, \psi\right)$ :

$$
\left(D_{\mathbb{S}^{1}} \psi, \psi\right)=\left(-h^{2} \psi^{\prime \prime}+i V \psi, \psi\right)=-h^{2}\left(\psi^{\prime \prime}, \psi\right)+i(V \psi, \psi)
$$


Учитывая, что $\left(\psi^{\prime \prime}, \psi\right)=-\left(\psi^{\prime}, \psi^{\prime}\right)$ (так как $\psi$ периодична), получаем

$$
\left(D_{\mathbb{S}^{1}} \psi, \psi\right)=h^{2}\left(\psi^{\prime}, \psi^{\prime}\right)+i(V \psi, \psi) .
$$

В правой части первое слагаемое действительное и неотрицательное, а второе чисто мнимое и для него легко сделать оценку (из теоремы о среднем, так как функция $\psi(x) \overline{\psi(x)}=|\psi(x)|^{2} \geqslant 0$ не меняет знак)

$$
\begin{aligned}
(V \psi, \psi) & =\int_{\mathbb{S}^{1}} V(x) \psi(x) \overline{\psi(x)} d x=\left.V(x)\right|_{x=x_{0} \in \mathbb{S}^{1}} \int_{\mathbb{S}^{1}} \psi(x) \overline{\psi(x)} d x \\
& \in\left[\min _{x \in \mathbb{S}^{1}} V(x), \max _{x \in \mathbb{S}^{1}} V(x)\right] \cdot\|\psi\|=[\min V, \max V] .
\end{aligned}
$$

Таким образом получаем, что

$$
\left\{\left(D_{\mathbb{S}^{1}} \psi, \psi\right) \mid \psi \in W_{2}^{2}\left(\mathbb{S}^{1}\right),\|\psi\|=1\right\} \subseteq[0,+\infty)+i[\min V, \max V]
$$

следовательно,

$$
\operatorname{PSP}_{h^{N}}\left(-h^{2} \frac{d^{2}}{d x^{2}}+i V(x)\right) \in \overline{[0,+\infty)+i[\min V, \max V]}=[0,+\infty)+i[\min V, \max V] .
$$

ЗАмечАниЕ 2. Для аналитичной $V(x) \not \equiv$ const числовой образ оператора $\mathfrak{D}-$ это полуполоса $[0,+\infty)+i(\min V, \max V)$. Действительно, в оценке для интеграла $(V \psi, \psi)$ равенство $\min V$ достигается только при $\left.V\right|_{\operatorname{supp} \psi}=\min V$. Так как $\psi$ непрерывна и не равна нулю в некоторой точке, это означает постоянство $V$ на некотором интервале. В этом случае аналитичная $V$ должна быть константой. То есть в оценке для интеграла $(V \psi, \psi)$ значение $\min V$ не достигается при $V \not \equiv$ const. Аналогично для $\max V$. При этом можно сколь угодно близко приблизиться к $\min V($ и $\max V$ ), беря в качестве $\psi$ функции, сосредоточенные в окрестности точки $\arg \min V$ (соответственно в окрестности точки $\arg \max V)$.

Обозначим $A_{V}=\left\{a \in \mathbb{R} \mid \exists x \in \mathbb{S}^{1}: V(x)=a, V^{\prime}(x) \neq 0\right\} ;$ Очевидно вложение $A_{V} \subseteq(\min V, \max V)$; кроме того, множество $(\min V, \max V) \backslash A_{V}$ конечно. Если бы оно было бесконечно, у аналитичной функции $V^{\prime}$ на компакте $\mathbb{S}^{1}$ было бы бесконечное число нулей. Тогда $V^{\prime} \equiv 0, V=$ const и $[\min V, \max V]=\{\cdot\}$, но одноточечное множество не может содержать бесконечное подмножество, таким образом, $A_{V}$ - это интервал $(\min V, \max V)$, из которого выкинуто конечное число точек (или $A_{V}=\varnothing$ для $V=$ const). Случай постоянного $V$ тривиален и мы его уже рассмотрели. В дальнейшем, будем считать что $V \not \equiv$ const.

На рис. 3 множество $(0,+\infty)+i A_{V}$ выделено серым цветом.

5. Явная конструкция почти собственных функций, отвечающих точкам полуполосы. Для любого натурального $N$ мы покажем, что $h^{N}$-псевдоспектр содержит множество $(0,+\infty)+i A_{V}-$ набор конечного числа полуполос, замыканием которого (в случае непостоянного $V$ ) является полуполоса $[0,+\infty)+i[\min V, \max V]$.

Лемма 3. Для любого $E \in(0,+\infty)+i A_{V}$ при любом натуральном $N$ существует функиия $\psi_{N}$ с нормой $\left\|\psi_{N}\right\|=1$, удовлетворяющая равенству (3), в котором $\varepsilon=h^{N}$ 


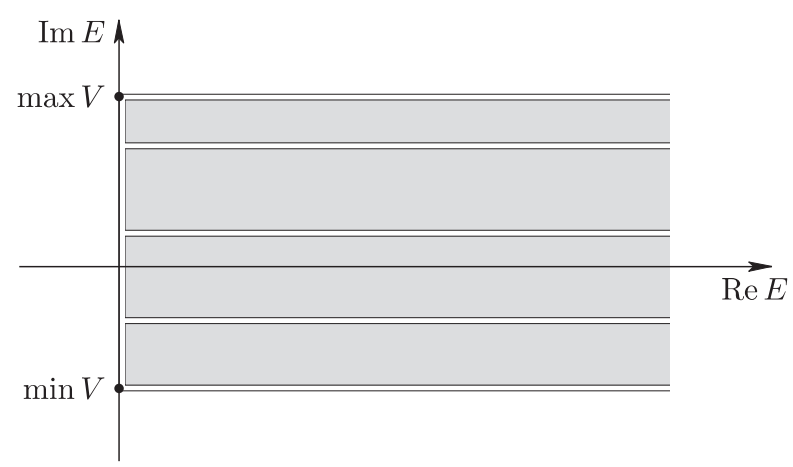

Рис. 3

ДоказАтЕЛьство. Зафиксируем произвольные $E \in(0,+\infty)+i A_{V}$ и $N \in \mathbb{N}$. Функцию $\psi_{N}$ построим в следующем виде:

$$
\psi_{N}(x)=e^{i S(x) / h} \sum_{\ell=0}^{N-2} h^{\ell} \chi_{\ell}(x) .
$$

Мы не будем писать индекс $N$ у функций $S$ и $\chi_{\ell}$ для того чтобы не загромождать запись; кроме того, отметим, что под $h^{0}$ подразумевается единица. При подстановке представления (4) в уравнение (3) получаем следующее условие:

$$
\begin{gathered}
\left(\left(S^{\prime}\right)^{2}+i V(x)-E\right) e^{i S(x) / h} \sum_{\ell=0}^{N-1} h^{\ell} \chi_{\ell}(x)-2 i S^{\prime} e^{i S(x) / h} \sum_{\ell=0}^{N-1} h^{\ell+1} \chi_{\ell}^{\prime}(x) \\
-i S^{\prime \prime} e^{i S(x) / h} \sum_{\ell=0}^{N-1} h^{\ell+1} \chi_{\ell}(x)-e^{i S(x) / h} \sum_{\ell=0}^{N-1} h^{\ell+2} \chi_{\ell}^{\prime \prime}(x)=O\left(h^{N}\right) .
\end{gathered}
$$

Это условие можно расписать по степеням $h$; тогда получим систему

$$
\left\{\begin{array}{l}
\left(\left(S^{\prime}\right)^{2}+i V(x)-E\right) e^{i S(x) / h} \chi_{0}=O\left(h^{N}\right), \\
\left(\left(S^{\prime}\right)^{2}+i V(x)-E\right) e^{i S(x) / h} \chi_{1}-\left(2 i S^{\prime} \chi_{0}^{\prime}-i S^{\prime \prime} \chi_{0}\right) e^{i S(x) / h}=O\left(h^{N-1}\right), \\
\left(\left(S^{\prime}\right)^{2}+i V(x)-E\right) e^{i S(x) / h} \chi_{\ell+1}-\left(2 i S^{\prime} \chi_{\ell}^{\prime}-i S^{\prime \prime} \chi_{\ell}+\chi_{\ell-1}^{\prime \prime}\right) e^{i S(x) / h}=O\left(h^{N-\ell-1}\right), \\
-\left(2 i S^{\prime} \chi_{N-2}^{\prime}-i S^{\prime \prime} \chi_{N-2}+\chi_{N-3}^{\prime \prime}\right) e^{i S(x) / h}=O(h), \\
\chi_{N-2}^{\prime \prime} e^{i S(x) / h}=O(1) .
\end{array}\right.
$$

Для удовлетворения системы (5) достаточно (нам необходимо лишь показать существование решения, а не найти их все) того, чтобы

$$
\left\{\begin{array}{l}
\left(\left(S^{\prime}\right)^{2}+i V(x)-E\right) e^{i S(x) / h}=O\left(h^{N}\right) \\
\chi_{\ell}(x) e^{i S(x) / h}=O(1) \\
\left(-2 i S^{\prime} \chi_{0}^{\prime}-i S^{\prime \prime} \chi_{0}\right) e^{i S(x) / h}=O\left(h^{N-1}\right), \\
\left(-2 i S^{\prime} \chi_{\ell}^{\prime}-i S^{\prime \prime} \chi_{\ell}\right) e^{i S(x) / h}=\chi_{\ell-1}^{\prime \prime} e^{i S(x) / h}+O\left(h^{N-\ell-1}\right), \\
\ell \in\{1, \ldots, N-1\} .
\end{array}\right.
$$


Для упрощения дополнительно сузим класс функций среди которых ищем $\psi_{N}$, a именно, будем искать их среди функций асимптотически (при $h \rightarrow 0)$ сосредоточенных в одной точке, т.е. функций, для которых

$$
\psi_{N}(x) \stackrel{h \rightarrow 0}{\longrightarrow} 0 \quad \text { при } \quad x \neq x_{0}
$$

при некотором $x_{0} \in \mathbb{S}^{1}$. Тогда $e^{i S(x) / h} \underset{h \rightarrow 0}{\rightarrow} 0$ при $x \neq x_{0}$ быстрее любой степени $h^{k}$, т.е.

$$
\operatorname{Im} S\left(\mathbb{S}^{1} \backslash\left\{x_{0}\right\}\right) \subseteq \mathbb{R}_{+}, \quad \operatorname{Im} S\left(x_{0}\right)=0 .
$$

Тогда $\operatorname{Im}\left(S^{\prime}\left(x_{0}\right)\right)=0$ и для выполнения первого условия системы $(6)$ возьмем $x_{0}$ и $S$ такие, что

$$
\left\{\begin{array}{l}
\operatorname{Re} E=\left(S^{\prime}\left(x_{0}\right)\right)^{2}, \\
\operatorname{Im} E=V\left(x_{0}\right) .
\end{array}\right.
$$

Это означает, что мы по заданной $E$ фиксируем точку $x_{0} \in \mathbb{S}^{1}$, в которой $V$ достигает значения $\operatorname{Im} E$ (и при этом, что понадобится нам в дальнейшем, $V^{\prime}\left(x_{0}\right) \neq 0$ ). Такая точка $x_{0}$ на окружности существует в силу определения множества $A_{V}$ и того, что $\operatorname{Im} E \in A_{V}$. Кроме того, мы также получили одно условие на функцию $S$.

Займемся построением функции $S$ в виде степенного ряда в точке $x_{0}$ :

$$
S(x)=\sum_{k \in \mathbb{Z}_{+}} S^{(k)}\left(x_{0}\right) \frac{\left(x-x_{0}\right)^{k}}{k !} .
$$

Так как искомая функция $\psi_{N}(x)$ определена условием (3) с точностью до ненулевого мультипликативного множителя, то $S\left(x_{0}\right)$ можно естественным образом выбирать произвольно из $\mathbb{C}$ при условии, что $e^{i S\left(x_{0}\right) / h} \neq 0$. Для определенности возьмем $S\left(x_{0}\right)=0$. Для того, чтобы норма $\psi_{N}$ была единична, в конце процедуры построения просто поделим $\psi_{N}$ на ее норму. Условие на $S^{\prime}\left(x_{0}\right)$ уже есть: $S^{\prime}\left(x_{0}\right)= \pm \sqrt{\operatorname{Re} E}$.

Функцию $V(x)$ представим в виде степенного ряда в точке $x_{0}$ (это можно сделать, так как $V(x)$ является аналитичной):

$$
V(x)=\sum_{k \in \mathbb{Z}_{+}} V^{(k)}\left(x_{0}\right) \frac{\left(x-x_{0}\right)^{k}}{k !},
$$

причем, как мы уже выяснили, $\operatorname{Im} E=V\left(x_{0}\right)$. Тогда условие $\left(S^{\prime}(x)\right)^{2}+i V(x)-E \equiv 0$ можно записать в виде (бесконечной) системы на $S^{k}\left(x_{0}\right), k \in \mathbb{N}$ :

$$
\left\{\begin{array}{l}
S^{(0)}\left(x_{0}\right)=0, \\
S^{(1)}\left(x_{0}\right)= \pm \sqrt{\operatorname{Re} E}, \\
i V^{k-1}\left(x_{0}\right)+\sum_{\substack{j, m \in \mathbb{N} \\
j+m=k+1}} \frac{S^{(j)}\left(x_{0}\right) S^{(m)}\left(x_{0}\right)}{(j-1) !(m-1) !}=0, \quad k \in \mathbb{N} \backslash\{1\} .
\end{array}\right.
$$

Хоть эта система и бесконечна, нас интересует лишь конечная ее часть. Действительно, для того, чтобы удовлетворить первое уравнение системы (6), не требуется точного $\left(S^{\prime}\right)^{2}+i V-E \equiv 0$, а достаточно чтобы $\operatorname{Re}\left(i S^{\prime}\left(x_{0}\right)\right)=0$ (это уже выполняется по второму уравнению полученной системы), $\operatorname{Re}\left(i S^{\prime \prime}\left(x_{0}\right)\right)<0$ (это требование 
придется добавить к системе) и $\left(S^{\prime}\right)^{2}+i V-E=O\left(\left(x-x_{0}\right)^{2 N}\right)$. Покажем это:

$$
\begin{aligned}
e^{i S(x) / h} O\left(\left(x-x_{0}\right)^{2 N}\right) & =e^{\operatorname{Re}(i S(x) / h)} e^{i \operatorname{Im}(\ldots)} O\left(\left(x-x_{0}\right)^{2 N}\right) \\
& =e^{\operatorname{Re}\left(i S^{\prime \prime}\left(x_{0}\right)\right)\left(x-x_{0}\right)^{2} / h+O\left(\left(x-x_{0}\right)^{3}\right)} O\left(\left(x-x_{0}\right)^{2 N}\right) .
\end{aligned}
$$

Теперь нужное свойство следует из очевидной оценки

$$
e^{\lambda\left(x-x_{0}\right)^{2} / h}\left(x-x_{0}\right)^{k}=e^{\lambda\left(x-x_{0} \sqrt{h}\right)^{2}}\left(x-x_{0} \sqrt{h}\right)^{k} \cdot h^{k / 2}=O\left(h^{k / 2+1 / 4}\right)
$$

при $\operatorname{Re} \lambda<0$ (оценка понимается в норме $\left.L_{2}\right)$. Таким образом, $e^{i S(x) / h} O((x-$ $\left.\left.x_{0}\right)^{2 N}\right)=O\left(h^{N}\right)$, значит, для выполнения первого равенства системы (6) нам нужно $\left(S^{\prime}\right)^{2}+i V-E=O\left(\left(x-x_{0}\right)^{2 N}\right)$ и, таким образом, нас интересует лишь следующая конечная часть выписанной ранее системы на $S^{(k)}\left(x_{0}\right)$ (с небольшой добавкой в виде неравенства):

$$
\left\{\begin{array}{l}
\operatorname{Re}\left(i S^{\prime \prime}\left(x_{0}\right)\right)<0, \\
S^{(0)}\left(x_{0}\right)=0, \\
S^{(1)}\left(x_{0}\right)= \pm \sqrt{\operatorname{Re} E}, \\
i V^{k-1}\left(x_{0}\right)+\sum_{\substack{j, m \in \mathbb{N} \\
j+m=k+1}} \frac{S^{(j)}\left(x_{0}\right) S^{(m)}\left(x_{0}\right)}{(j-1) !(m-1) !}=0, \quad k \in \mathbb{N} \cap[2,2 N-1] .
\end{array}\right.
$$

Последнее уравнение этой системы можно переписать:

$$
\frac{2}{(k-1) !} S^{(1)}\left(x_{0}\right) S^{(k)}\left(x_{0}\right)=-i V^{(k-1)}\left(x_{0}\right)-\sum_{\substack{j, m \in \mathbb{N} \backslash\{1\} \\ j+m=k+1}} \frac{S^{(j)}\left(x_{0}\right) S^{(m)}\left(x_{0}\right)}{(j-1) !(m-1) !},
$$

значит, (так как $S^{(1)}\left(x_{0}\right)= \pm \sqrt{\operatorname{Re} E} \neq 0$ ) из этой системы однозначно (при фиксированном знаке $\left.S^{\prime}\left(x_{0}\right)\right)$ находятся все $S^{(k)}$; таким образом находится $S$. Остается только выполнить добавочное требование $\operatorname{Re}\left(i S^{\prime \prime}\left(x_{0}\right)\right)<0$. Из последнего уравнения системы при $k=2$ имеем $S^{\prime \prime}\left(x_{0}\right)=-i V^{\prime}\left(x_{0}\right) /\left(2 S^{\prime}\left(x_{0}\right)\right)$; значит, добавочное требование можно удовлетворить подходящим выбором (произвольного до этого момента) знака $S^{\prime}\left(x_{0}\right)$, если только $V^{\prime}\left(x_{0}\right) \neq 0$. Именно для этого и требовалось вводить множество $A_{V}$ и выбирать $x_{0}$ с оглядкой на $V^{\prime}\left(x_{0}\right) \neq 0$. Итак, мы определились со знаком $S^{\prime}\left(x_{0}\right)$ :

$$
S^{\prime}\left(x_{0}\right)=-\operatorname{sign}\left(V^{\prime}\left(x_{0}\right)\right) \sqrt{\operatorname{Re} E} .
$$

Таким образом, найдя из системы $(8) S^{(k)}$ при $k<2 N$ и полагая $S^{(k)}\left(x_{0}\right)=0$ при $k \geqslant 2 N$, мы однозначно построили $S$ в виде многочлена по $\left(x-x_{0}\right)$. При этом мы удовлетворили и первое и второе уравнение системы (6).

Теперь займемся построением $\chi_{\ell}(x)$. Для того, чтобы выполнялись третье и четвертое уравнение системы (6), достаточно (по построению $S$ ) выполнения

$$
\left\{\begin{array}{l}
-2 i S^{\prime} \chi_{0}^{\prime}-i S^{\prime \prime} \chi_{0}=0 \\
-2 i S^{\prime} \chi_{\ell}^{\prime}-i S^{\prime \prime} \chi_{\ell}=\chi_{\ell-1}^{\prime \prime}, \quad \ell \in\{1, \ldots, N-1\}
\end{array}\right.
$$

в некоторой окрестности $U^{\prime}\left(x_{0}\right)$ точки $x_{0}$. Так как по построению $S^{\prime}\left(x_{0}\right) \neq 0$, окрестность $U^{\prime}\left(x_{0}\right)$ можно выбрать таким образом, что на ней $S^{\prime}$ отделена от нуля. Тогда находим $\chi_{0}$ :

$$
2 S^{\prime} \chi_{0}^{\prime}+S^{\prime \prime} \chi_{0}=0 \Longleftrightarrow \chi_{0}=\operatorname{const}\left(S^{\prime}\right)^{-1 / 2}
$$


Мультипликативную константу в формуле для $\chi_{0}$ можно выбирать произвольно среди ненулевых чисел. Для определенности положим ее равной единице:

$$
\chi_{0}=\left(S^{\prime}\right)^{-1 / 2} .
$$

Затем из уравнения

$$
2 S^{\prime} \chi_{1}^{\prime}+S^{\prime \prime} \chi_{1}=i \chi_{0}^{\prime \prime}=i \frac{3 S^{\prime \prime}-2 S^{\prime} S^{\prime \prime \prime}}{4\left(S^{\prime}\right)^{5 / 2}},
$$

находим $\chi_{1}$, и так далее. Так как $S^{\prime}$ отделена от нуля на $U^{\prime}\left(x_{0}\right)$, решение уравнения

$$
\chi_{\ell}^{\prime}=\frac{-S^{\prime \prime} \chi_{\ell}+i \chi_{\ell-1}^{\prime \prime}}{2 S^{\prime}}
$$

в окрестности $U^{\prime}\left(x_{0}\right)$ существует и гладкое.

Построенная таким образом $\psi_{N}$ обладает лишь одним недостатком - она не периодична. Но это легко устранить. Домножим ее на гладкую срезающую функцию, равную единице в некоторой окрестности точки $x_{0}$ и нулю вне другой окрестности этой точки. Теперь можно просто переопределить $\psi_{N}$ так, чтобы она была периодична - просто возьмем вместо нее сумму (эта сумма в каждой точке $x$ конечна)

$$
\sum_{k \in \mathbb{Z}} \psi_{N}(x+k T)
$$

Осталось только поделить $\psi_{N}$ на ее ненулевую (так как $\psi_{N}$ непрерывна и $\psi_{N} \not \equiv 0$ ) норму в $L_{2}\left(\mathbb{S}^{1}\right)$ и мы завершим доказательство.

Теорема 3. В случае непостоянной $V(x)$ для любого $N$ из натурального ряда $h^{N}$-псевдоспектр оператора $-h^{2} d^{2} / d x^{2}+i V(x)$, заданного на окружности, равен полуполосе $[0,+\infty)+i[\min V, \max V]$.

ДоказАтельство. Как уже отмечалось, замыканием множества $(0,+\infty)+i A_{V}$ является полуполоса $[0,+\infty)+i[\min V, \max V]$ (в случае непостоянного $V$ ). Значит, для доказательства достаточно объединить результаты лемм 1,3 и следствия 1.

В работе [8] анонсирована экспоненциальная оценка скорости роста резольвенты аналогичного оператора (с граничными условиями на отрезке вместо условия периодичности) в полуполосе числового образа.

\section{СПИСОК ЦИТИРОВАННОЙ ЛИТЕРАТУРЫ}

[1] S. Yu. Dobrokhotov, V. N. Kolokoltsov, V. M. Olivé, "Quasimodes of the diffusion operator $-\varepsilon \Delta+v(x) \cdot \nabla$, corresponding to asymptotically stable limit cycles of the field $v(x)$ ", Third Symposium on Probability Theory and Stochastic Processes (Hermosillo, 1994), Aportaciones Mat. Notas Investigación, 11, Soc. Mat. Mexicana, México, 1994, 81-89.

[2] С. Ю. Доброхотов, В.Н. Колокольцов, В. Мартинес Оливе, "Асимптотически устойчивые инвариантные торы векторного поля $V(x)$ и квазимоды оператора диффузии", Матем. заметки, 58:2 (1995), 301-306.

[3] С. А. Степин, "Несамосопряженные сингулярные возмущения и спектральные свойства задачи Орра-Зоммерфельда", Матем. сб., 188 (1997), 129-146.

[4] А. А. Шкаликов, "О предельном поведении спектра при больших значениях параметра одной модельной задачи”, Матем. заметки, 62:6 (1997), 950-953. 
[5] С. А. Степин, А.А. Аржанов, "О локализации спектра в одной задаче сингулярной теории возмущений", УМН, $\mathbf{5 7 : 3 ~ ( 2 0 0 2 ) , ~ 1 6 1 - 1 6 2 . ~}$

[6] С.Н.Туманов, А. А. Шкаликов, "О предельном поведении спектра модельной задачи для уравнения Орра-Зоммерфельда с профилем Пуазейля", Изв. РАН. Сер. матем., 66:4 (2002), 177-204.

[7] С. В. Гальцев, А.И. Шафаревич, "Асимптотика дискретного спектра несамосопряженного периодичного оператора.", Труды XXVII Конферениии молодых ученых, Издво мех.-мат. фак-та МГУ, М., 2005, 18-22.

[8] A. A. Schkalikov, "Spectral portraits and the resolvent growth of a model problem associated with the Orr-Sommerfeld equation", arXiv: math.FA/0306342v1.

С. В. Гальцев, А. И. Шафаревич

Поступило

Московский государственный университет

14.12 .2005

им. М. В. Ломоносова

Исправленный вариант

E-mail: galtsev@mccme.ru, shafar@mech.math.msu.su

16.03 .2006 